Annuaire suisse de politique de développement

25-2 | 2006

Paix et sécurité : les défis lancés à la coopération internationale

\title{
Les conflits liés aux ressources naturelles. Résultats de recherches et perspectives
}

Laurent Goetschel et Didier Péclard

\section{OpenEdition}

\section{Journals}

Édition électronique

URL : http://journals.openedition.org/aspd/255

DOI : 10.4000/aspd.255

ISSN : 1663-9669

Éditeur

Institut de hautes études internationales et du développement

Édition imprimée

Date de publication : 1 octobre 2006

Pagination : 95-106

ISBN : 2-88247-064-9

ISSN : $1660-5934$

Référence électronique

Laurent Goetschel et Didier Péclard, « Les conflits liés aux ressources naturelles. Résultats de recherches et perspectives ", Annuaire suisse de politique de développement [En ligne], 25-2 | 2006, mis en ligne le 18 décembre 2009, consulté le 07 septembre 2020. URL : http://journals.openedition.org/ aspd/255 ; DOI : https://doi.org/10.4000/aspd.255 


\title{
Les conflits liés aux ressources naturelles
}

Résultats de recherches et perspectives

\author{
Laurent Goetschel* et Didier Péclard**
}

L

'histoire des liens entre ressources naturelles et conflits est du domaine de la longue durée. Depuis des siècles, sociétés et Etats ont utilisé certaines ressources naturelles afin de promouvoir leurs intérêts et de poursuivre leurs objectifs politiques. Jusqu'à la fin du XVIII ${ }^{\mathrm{e}}$ siècle par exemple, le bois a été d'une importance primordiale pour les puissances navales. De nos jours, c'est le pétrole qui fait la une des médias internationaux, aussi bien comme élément indispensable à la politique des grandes puissances que comme source de conflit. Il est question d'une véritable géopolitique des conflits liés aux ressources naturelles ${ }^{1}$. Mais les Etats n'ont pas le monopole de l'appropriation de celles-ci à des fins politiques ou militaires. Le contrôle sur les ressources naturelles ou leur utilisation est également un facteur important de tensions, voire de conflits entre groupes sociaux, qu'il s'agisse de ressources non renouvelables comme les diamants, ou de ressources renouvelables telles que l'eau ou la terre.

$\mathrm{Si}$ anciens soient-il, les liens entre ressources naturelles et conflits n'en demeurent pas moins problématiques, et ils demandent à être interrogés de façon critique. C'est ce à quoi se sont attachées les recherches menées sur cette thématique dans le cadre du $N C C R^{2}$ North-South et plus précisément au sein du Individual Project (IP) 7 piloté par la Fondation suisse pour la paix (Swisspeace) $)^{3}$, auquel se sont associés également des chercheurs du Centre de politique de sécurité (CSS) ${ }^{4}$ de l'EPFZ et du Séminaire d'ethnologie de l'Université de Zurich (ESZ) ${ }^{5}$. Ces recherches se sont situées dans la suite des travaux pionniers menés également au sein de Swisspeace et du css par Günther Baechler et Kurt Spillmann pour le projet Environmental Change and Conflict (ENCOP) ${ }^{6}$. Dans les pages qui suivent, nous commençons par replacer ces recherches dans leur contexte politique et historiographique, puis nous présentons de manière succincte leurs principaux résultats. En outre, nous montrons, sur la base notamment de différentes études de cas réalisées par des doctorant·e.s de l'IP7, que l'idée largement répandue dans la

* Coordinateur du programme Gouvernance et conflit, Fondation suisse pour la paix (Swisspeace), Berne.

** Directeur de la Fondation suisse pour la paix (Swisspeace), Berne.

1 P. Le Billon, «The Geopolitical Economy of "Resource Wars" », dans Geopolitics of Resource Wars, Ph. Le Billon (ed.), London; New York, Frank Cass, 2005, pp. 1-28.

2 National Centre of Competence in Research ou Pôle de recherche national (PRN). Pour des détails sur ce pôle, voir <http://www.nccr-north-south.unibe.ch>.

$<$ http://www.swisspeace.org $>$.

$<$ http://www.css.ethz.ch>.

$<$ http://www.ethno.unizh.ch $>$.

6 G. Baechler, V. Böge, S. Klötzli, S. Libiszewski, K.R. Spillmann, Kriegsursache Umweltzerstörung. Ökologische Konflikte in der Dritten Welt und Wege ihrer friedlichen Bearbeitung (ENCOP Band I), Zürich; Chur, Rüegger, 1996. 
littérature scientifique selon laquelle il y aurait un lien de causalité directe entre environnement et conflits n'a pas réellement de fondement empirique. De même, la portée heuristique de cette vision classique doit être remise en cause: en se concentrant sur ces liens de causalité environnementale, le risque est grand de ne pas donner la place qui leur revient aux véritables enjeux historiques, politiques et économiques de ces conflits. Nous dégageons enfin quelques pistes de recherche pour développer la réflexion sur ce sujet.

De la " sécurité environnementale " à la " gestion des ressources naturelles"

Vers la fin de la guerre froide, le concept de «sécurité environnementale» est apparu aux niveaux aussi bien international que national comme produit de coïncidences d'ordres divers. Les sécheresses et la désertification rapide du Sahel, l'accident de la centrale nucléaire de Tchernobyl en 1986, puis les débats sur la mort des forêts en Europe de l'Ouest ont ainsi largement contribué à rendre le grand public attentif et sensible aux «menaces» induites par notre environnement. Il est dès lors apparu possible, en effet, que le bien-être des populations se voie affecté par la détérioration de l'environnement, détérioration causée directement ou indirectement par l'activité humaine et par le «changement climatique» qu'elle avait provoqué. Sur le plan politique, la fin de la guerre froide et l'ouverture du débat sur les «nouveaux défis sécuritaires» ont élargi l'éventail des menaces. La sécurité des Etats ne dépendait désormais non plus seulement de la défense contre les missiles nucléaires, mais également de la protection de l'environnement ${ }^{7}$. Des organisations militaires de défense telles que l'OTAN ont mis sur pied des forums de réflexion sur les menaces environnementales. Et des think tanks américains établis, à l'image du Woodrow Wilson Center, ont créé des programmes spéciaux voués à l'étude de ce genre de conflits ${ }^{8}$. La question était de savoir quand et sous quelles conditions l'environnement risquait de devenir une «menace». Cependant, dans cette foison d'initiatives diverses et de réflexions, il était difficile de distinguer quelle était, de fait, la préoccupation centrale entre protection de l'environnement, notre propre sécurité, ou encore celle des pays d'où provenaient certaines des ressources naturelles en question'.

C'est sur cette toile de fond que différents projets de recherche ont été lancés au début des années 1990 pour étudier les liens éventuels entre diminution de l'offre de ressources naturelles par rapport à leur demande et développement de conflits de type violent. Il s'agit notamment d'un projet canadien dirigé par Thomas Homer-Dixon ${ }^{10}$ et du projet suisse précité, sous la direction de Günther Baechler, tous deux basés sur un échantillon important d'études de cas ${ }^{11}$. L'hy-

7 B. Buzan, People, States, and Fear: An Agenda for International Security Studies in the Post-cold War Era, Boulder, CO, Lenne Rienner, 1991; J.T. Mathews, «Redefining Security», Foreign Affairs, vol. 68, nº 2, 1989, pp. 162-177.

8 Environmental Change and Security Project (ECSP), the Woodrow Wilson International Center for Scholars, <http://www.wilsoncenter.org/ecsp>.

9 L. Brock, «Umwelt und Konflikt im internationalen Forschungskontext», dans Umwelt und Sicherheit. Herausforderungen für die internationale Politik, A. Carius, K.M. Lietzmann (eds.), Berlin, Springer, 1998, pp. 37-56.

10 T.F. Homer-Dixon, Environment, Scarcity, and Violence, Chichester, Princeton, 1990.

11 Pour l'ouvrage de synthèse le plus systématique du deuxième projet, voir G. Baechler, Violence through Environmental Discrimination, Dordrecht; Boston; London, Kluwer Academic Publishers, 1999. 
pothèse de base des deux équipes stipulait une causalité entre pénurie accrue de ressources naturelles (increased scarcity) et une augmentation de la probabilité de conflits violents entre les populations directement concernées.

En résumé, les résultats de ces travaux sont plutôt nuancés: Günther Baechler et Thomas Homer-Dixon ont démontré que la destruction et la pénurie de ressources naturelles renouvelables ne représentent que très rarement une cause suffisante et immédiate de conflits violents ${ }^{12}$. Mais tous deux ajoutent que ces phénomènes peuvent par contre souvent contribuer de manière importante à de tels conflits lorsqu'ils s'accompagnent d'autres facteurs tels que des problèmes économiques, sociaux ou ethniques - facteurs dont l'incidence est généralement plus forte dans des pays en voie de développement que dans d'autres. Peu à peu, un consensus s'est fait autour de l'idée que les conflits liés à l'environnement renvoient à une pluralité de causes, qu'ils se caractérisent par leur réciprocité (la dégradation de l'environnement comme cause de conflit et vice-versa) et par le fait qu'un conflit n'est qu'une conséquence parmi d'autres de la dégradation de l'environnement - la migration, la pauvreté ou encore l'insécurité alimentaire représentant d'autres conséquences typiques ${ }^{13}$.

C'est sur cette base historiographique qu'ont été lancées en 2001 les recherches menées au sein de l'IP7, à savoir le programme de recherche individuel du Pôle de recherche Nord-Sud voué à l'étude des conflits liés à l'environnement ${ }^{14}$. A la différence toutefois des travaux menés par les équipes de Günther Baechler et de Thomas Homer-Dixon, les recherches de l'IP7 ne se sont pas fixé comme objectif premier d'ouvrir à nouveau le débat sur les liens de causalité entre ressources naturelles et conflits - même si ce débat a bien entendu été pris en compte dans nos recherches ${ }^{15}$. L'analyse des conflits liés à l'environnement telle que développée à l'IP7 devait plutôt servir à comprendre les conditions de leur transformation, voire à suggérer des pistes en vue de leur résolution. En évitant de se cantonner dans l'étude des causalités entre ressources et conflits, l'IP7 a également pris en compte l'analyse de situations où des tensions, voire des conflits latents au sujet de certaines ressources naturelles n'avaient pas débouché sur des conflits violents et déclarés. Encore une fois, il s'agissait de mettre l'accent non pas sur des causalités de différents degrés entre environnement et conflits, mais plutôt sur les modes de gestion ou de traitement de telles situations conflictuelles.

Cette perspective axée sur la transformation des conflits était directement liée au cadre conceptuel global du Pôle de recherche Nord-Sud, dont l'objectif prioritaire est d'accroître et d'améliorer le savoir scientifique sur la prévention des conflits liés à ce que l'on appelle, dans ce cadre, des "syndromes du changement global ${ }^{16}$. Suivant cette approche, des «nœuds» (clusters) de problèmes

12 A. Carius, G. Baechler, S. Pfahl, A. March, F. Biermann, Umwelt und Sicherheit: Forschungserfordernisse und Forschungsprioritäten, Berlin, Ecologic, 1999.

13 Ibid., p. 5.

14 Voir la page <http://www.swisspeace.org/research/governance_conflict_env.htm>. Pour une liste des thèses achevées et en cours, voir <http://www.swisspeace.org/uploads/research/DoctoralThesis8June06. pdf $>$.

15 Pour une mise en perspective critique de ce débat, voir T. Hagmann, «Confronting the Concept of Environmentally Induced Conflict», Peace, Conflict and Development, $\mathrm{n}^{\circ}$ 6, January 2005, pp. 1-22.

16 Pour l'approche méthodologique ayant servi de base à la conception du programme de recherche, voir les trois premiers chapitres de l'ouvrage suivant: H. Hurni, U. Wiesmann, R. Schertenleib (eds.), Research for Mitigating Syndromes of Global Change: A Transdisciplinary Appraisal of Selected 
ont tendance à se reproduire dans des configurations semblables dans différentes parties du monde. Les problèmes en question sont interconnectés et on ne peut y remédier qu'en tenant compte des différentes composantes du «syndrome » auquel ils sont liés. Par rapport à la transformation des conflits liés aux ressources naturelles, cela implique que les recherches pensent «large» tant par rapport aux causes ou aux facteurs de tels conflits qu'en ce qui concerne les moyens possibles d'y remédier.

Pour éviter de nous enfermer dans des débats sans issue certaine sur l'importance de l'environnement comme facteur de conflits, un pas supplémentaire nous est rapidement apparu comme incontournable. Ce pas a consisté à mettre au centre de nos préoccupations et de notre problématique la question de la gestion des ressources naturelles. Ainsi, dans la perspective que nous avons adoptée, les «conflits environnementaux » sont perçus comme des conflits autour de l'utilisation de ressources naturelles renouvelables, et nous considérons qu'il y a situation conflictuelle lorsque l'un au moins des acteurs est lésé par les divergences de position entre les parties impliquées dans le conflit. Avec cette définition, nous nous sommes clairement positionnés dans ce que l'on a appelé la «troisième phase» des recherches sur les conflits liés à l'environnement: après une première phase où la préoccupation centrale avait été d'élargir au niveau conceptuel la notion de sécurité, une deuxième qui se caractérisait par l'accent mis sur les liens de causalité entre environnement et conflit, il s'agissait désormais de se concentrer sur les modes de gestion adaptés à ce type de situation conflictuelle et ayant pour objectif la transformation desdits conflits afin d'éviter qu'ils ne débouchent sur une spirale de violence ${ }^{17}$.

Interdisciplinarité, comparatisme et tandems Nord-Sud

Le programme a suivi une approche interdisciplinaire et comparative, basée sur un nombre limité d'études de cas approfondies. L'interdisciplinarité est un élément essentiel de toute recherche sur la paix et les conflits. En effet, tout comme les dimensions de la paix ne peuvent être réduites à un seul domaine tel que l'économie, la politique ou l'ordre juridique, les causes de conflits sont elles également d'ordres divers. Ainsi, l'IP7 a regroupé des chercheurs provenant de disciplines comme la géographie, l'ethnologie, la science politique ou encore l'économie, et des étudiant·e·s de niveau master formé·e·s dans d'autres disciplines sont venu·e.s encore élargir le spectre d'approches prises en compte. Le dialogue entre les différentes recherches, par-delà les frontières disciplinaires, a été intense et continu.

L'approche comparative, quant à elle, s'est inscrite dans la stratégie de recherche développée au sein du NCCR North-South, stratégie qui stipulait l'existence de clusters de problèmes de développement liés à des contextes géographiques spécifiques ${ }^{18}$. Les travaux de 1'IP7 se sont concentrés sur quatre des neuf

Regions of the World to Prepare Development-Oriented Research Partnerships. Perspectives of the Swiss National Centre of Competence in Research (NCCR) North-South, University of Berne, vol. 1. Bern, Geographica Bernensia, 2004.

17 A. Carius et al., op. cit., pp. 12-14.

18 H. Hurni et al., op. cit. Voir la page <http://www.nccr-north-south.unibe.ch/syndromeliste.asp> 
Joint Areas of Case Studies (JACS) du NCCR Nord-Sud ${ }^{19}$, soit la Corne de l'Afrique (Ethiopie, Soudan et Egypte), l'Asie centrale (Ouzbékistan, Tadjikistan et Kirghizistan), l'Asie du Sud (Inde et Bangladesh) ainsi que l'Asie du SudEst (Philippines et Indonésie). D'autre part, l'IP7 a mis en place une structure dite de tandem pour ses doctorant.e.s provenant de pays du Nord et du Sud ou de l'Est. Celle-ci consistait à conduire des études de cas dans le même contexte géographique par des chercheurs d'origines différentes, l'objectif étant de favoriser l'échange de connaissances et l'intégration de chercheurs provenant de pays du Sud dans les réseaux académiques internationaux. En outre, l'IP7 a opté pour une douzaine d'études de cas approfondies, étant entendu que les questions posées par ces recherches individuelles étaient censées apporter des résultats potentiellement applicables à d'autres cas. Toutefois, les chercheurs ont été encouragés à choisir un angle de vue qui déborde le cadre strict de leur contexte de recherche spécifique, et qui ne se limite pas à vouloir corroborer ou critiquer une théorie ou une approche préexistante. L'objectif était de mettre le mieux possible en valeur les nouvelles informations et les nouvelles interprétations pouvant ressortir des études de terrain et de contribuer ainsi à une réponse aux questions posées ${ }^{20}$.

D'un point de vue méthodologique, nous avons procédé sur les modes aussi bien inductif que déductif. Nous avons cherché autant à élaborer des instruments pratiques tirés de cadres théoriques préexistants qu'à développer et à tester des instruments préexistants dans de nouvelles constellations de conflits. Les résultats empiriques produits nous ont permis d'enrichir et de faire progresser la réflexion conceptuelle et théorique en la matière. En conséquence, la palette d'outils méthodologiques utilisés s'est avérée assez large, allant de la recherche sur la base d'indicateurs sociaux, politiques ou économiques à des enquêtes de terrain ethnologiques «classiques», en passant par la récolte de données sur le terrain ou dans des archives.

\section{Pour une approche anthropocentrée des conflits environnementaux}

Les résultats présentés ci-dessous se basent sur des recherches achevées ou en cours, toutes les thèses de doctorat lancées dans le cadre de ce programme n'ayant pas encore été soumises. L'heure n'est donc pas encore au bilan final. Néanmoins, ces résultats permettent à notre avis de tirer des premières conclusions, aussi bien par rapport aux études de cas spécifiques que par rapport aux travaux de recherche préexistants. Dans les différentes régions traitées, l'accent des recherches a, d'un point de vue thématique, été mis d'une part sur les conflits internationaux liés à la gestion de l'eau, et, de l'autre, sur les conflits nationaux (ou subnationaux) liés à l'accès à la terre et à sa distribution.

19 Le NCCR Nord-Sud se concentre sur neuf régions du monde (JACS), l'une en Suisse et les huit autres en Afrique, en Amérique latine et en Asie. Voir la page <http://www.nccr-north-south.unibe.ch/Jacsliste. asp>.

20 Un bon aperçu du potentiel scientifique et heuristique d'études de cas est donné dans J. Gerring, «What Is a Case Study and What Is It Good For?», American Political Science Review, vol. 98, n 2 , 2004, pp. 341-354. 


\section{Eau et politique}

La gestion de l'eau dans le bassin du Nil, qui s'étend sur une dizaine de pays et compte environ 160 millions d'habitants, représente un potentiel important de conflit. Les eaux du plus long fleuve du monde sont en effet une ressource essentielle pour l'agriculture et l'économie de pays situés pour la plupart dans des zones arides ou semi-arides. Avec un taux de croissance annuel de $2 \%$ à $3 \%$, la population de la région exerce une pression croissante sur cette ressource, et le risque d'un déséquilibre grandissant entre l'offre et la demande en eau est réel. De plus, des intérêts opposés entre pays riverains viennent compliquer la donne: ainsi, entre l'Ethiopie, dont les hauts plateaux fournissent $86 \%$ des eaux du Nil, et l'Egypte, à qui le fleuve fournit $95 \%$ de ses besoins en eau, les relations ont été parfois très tendues ${ }^{21}$.

Sur cette thématique, deux recherches conjointes ont été menées, l'une en amont du fleuve (Ethiopie) ${ }^{22}$ et l'autre en aval (Egypte ${ }^{23}$, sur la manière dont on pouvait passer «du conflit à la coopération» (Simon Mason) et ainsi résoudre le «dilemme hydropolitique» entre, d'une part, une gestion non adéquate et un usage contraire aux principes du développement durable à l'échelon national, et, de l'autre, le manque de coopération et l'insécurité régnant dans la région ${ }^{24}$. Les deux auteurs ont montré toute l'importance des stratégies dites de linkage («liaison») dans la gestion de l'eau et dans la prévention des conflits liés à cette gestion problématique: liens entre pays riverains dont les destins sont immanquablement interdépendants de par leur situation géographique et qui ont tout à gagner à collaborer entre eux; liens institutionnels, économiques, environnementaux entre tous les protagonistes du bassin. Contre une forte tendance historique à des approches unilatérales de la gestion et de l'utilisation des eaux du bassin du Nil et afin de gérer de manière constructive les conflits liés à cette utilisation, ces recherches suggèrent que toute extraction d'eau soit compensée par la mise à disposition d'autres ressources ou par l'octroi d'une «voix au chapitre», c'est-à-dire la participation politique des populations défavorisées au processus de décision et de gestion des ressources. La mise en œuvre de tels mécanismes de compensation et la prise en compte des populations concernées présupposent la création de mécanismes institutionnels adéquats. Ceux-ci doivent transgresser les frontières nationales et tenir compte de mécanismes de pouvoir aussi bien nationaux que traditionnels au niveau régional ${ }^{25}$.

21 S.A. Mason, From Conflict to Cooperation in the Nile Basin: Interaction between Water Availability, Water Management in Egypt and Sudan, and International Relations in the Eastern Nile Basin, Zurich, Center for Security Studies, ETH Zurich, 2004, p. 3.

22 Y. Arsano, Ethiopia and the Nile: Dilemmas of National and Regional Hydropolitcs, PhD Thesis, Department of Political Science and International Relations, Addis Ababa, Addis Ababa University, 2004

23 S.A. Mason, op. cit. Yacob Arsano, du Département de science politique de l'Université d'AddisAbeba, et Simon Mason, du Centre for Security Studies de l'EPFZ, ont développé ensemble une méthode originale de travail basée sur des ateliers de discussion (dialogue workshops) et élaboré leurs recherches en tandem Nord-Sud.

24 Y. Arsano, Ethiopia and the Nile: Dilemma of National and Regional Hydro-politics, PhD Summary, NCCR North-South Dialogue, IP 7 «Environmental Change and Conflict Transformation», Bern, NCCR North-South, 2005, <http://www.nccr-north-south.unibe.ch/document/document.asp?ID=3741\&refTitle= the $\% 20$ NCCR $\% 20$ North-South $\&$ Context=NCCR $>$.

25 Dans une perspective de recherche plus directement appliquée, voir R. Bonzi, «NGOs in Conflict Prevention: Experiences from the Water Sector in Ethiopia», Development in Practice, vol. 16, $\mathrm{n}^{\circ} 2$, 2006, pp. 201-208. 
La question de l'eau est également un enjeu économique, politique et social très important en Asie centrale. Là encore, une tendance lourde, constatée aussi bien dans la recherche scientifique que dans des projets de développement, consiste à établir un lien direct entre ressources en eau et conflits. Le manque (scarcity) d'eau y est généralement perçu comme la cause «naturelle» de doléances (grievances) qui, une fois formulées et portées dans l'arène publique par les communautés locales, mènent presque immanquablement à des conflits violents. Dans cette perspective, les conflits liés à l'eau sont considérés comme «endémiques », c'est-à-dire comme résultant seulement de luttes et d'enjeux à l'échelon local, ou comme une conséquence directe d'une dégradation des relations intercommunautaires due au manque d'eau. Et la résolution de ces conflits est perçue avant tout comme un problème à la fois technique (amélioration des réseaux d'irrigation) et local (mise sur pied de mécanismes de cogestion de l'eau à l'échelon communautaire).

Les recherches menées au sein de l'IP7 sur la vallée de Ferghana, dans le bassin du Syr Daria aux confins du Kirghizistan, de l'Ouzbékistan et du Tadjikistan ${ }^{26}$, ont bien montré les limites d'une telle approche. Le concept même de «manque» d'eau tout d'abord n'est pas une simple donnée «objective» ou «naturelle», comme cela est généralement postulé, notamment par des agences de développement actives dans la région. Au contraire, le manque d'eau est un problème historiquement, socialement, culturellement et politiquement construit. Il est la résultante de rapports de force, et la manière dont les acteurs sociaux perçoivent les problèmes d'approvisionnement en eau potable ou d'irrigation, ainsi que les stratégies qu'ils mettent (ou ne mettent pas!) en œuvre pour résoudre ces problèmes doivent donc être contextualisées. De plus, analyser ces conflits comme «endémiques» dans les sociétés concernées pose problème. D'une part, cela débouche généralement sur une vision primordialiste et homogénéisante de ces communautés, perçues comme des entités homogènes formées autour de liens de solidarité essentialistes et poursuivant un objectif commun, alors même qu'il s'agit de sociétés politiques complexes parcourues de multiples lignes de fracture. D'autre part, ces conflits ne ressortissent pas seulement à des logiques locales; au contraire, on ne peut les comprendre si on ne les replace pas dans leur contexte international, et si l'on ne perçoit pas les enjeux politiques qui se posent au niveau régional.

Du fait de la vision positiviste des liens entre scarcity, grievances et conflits exposée plus haut, les réponses apportées par les acteurs nationaux et internationaux au problème de la répartition de l'eau dans la vallée de Ferghana ne sont souvent pas adéquates. Tout d'abord, l'option «techniciste», qui consiste à tenter de prévenir ou de résoudre les conflits liés à l'eau en améliorant les réseaux de distribution et leur gestion par les communautés locales, a pour principal défaut qu'elle tend à «dépolitiser» des problèmes qui sont hautement politiques en en faisant de «simples» questions de développement technique et communautaire ${ }^{27}$. La prise en compte d'une ressource naturelle telle que l'eau comme

26 C. Bichsel, Dangerous Divides: Irrigation Disputes and Conflict Transformation in the Ferghana Valley, PhD thesis, Bern, Institute of Geography, University of Bern, 2006.

27 Dans un contexte tout à fait différent, voir aussi J. Ferguson, The Anti-Politics Machine: «Development», Depoliticization, and Bureaucratic Power in Lesotho, Minneapolis, University of Minnesota Press, 1990. 
étant un problème avant tout politique, social et culturel s'avère donc être un enjeu prioritaire, aussi bien de la recherche que des politiques de développement.

Ensuite et par voie de conséquence, le rôle, pourtant primordial, des rapports de force au niveau local tend à être sous-estimé, et il n'est pas non plus suffisamment pris en compte dans la mise en œuvre des programmes d'agences internationales actives dans la région. Ces rapports de force se reflètent dans l'importance relative accordée aux différents systèmes de loi, que ce soit à l'échelon national, régional ou local. Or, bien souvent, le cadre juridique national, retravaillé et réformé avec l'aide d'acteurs internationaux, est en décalage complet avec la vie quotidienne des populations locales, et il ne fait donc pas réellement sens pour elles. De plus, les groupes concernés ne partagent pas ou ne se reconnaissent pas dans le modèle d' ' harmonie » que des intervenants externes tentent d'imposer comme modèle de solutions des conflits qu'ils ont cru repérer. Ce genre de modèle est souvent d'ailleurs une projection symbolique de la part des donateurs sans réel fondement historique. Des conflits qui, en apparence, résultent «simplement» de la compétition pour l'accès à l'eau et aux réseaux d'irrigation résultent en effet de l'histoire des rapports de force entre différents groupes constitués et couches sociales. La transformation des conflits liés à l'eau ne peut donc faire l'économie d'une analyse approfondie de leurs dynamiques historiques, sociales, culturelles et politiques, ce qui n'est souvent pas dans l'intérêt ni des acteurs internationaux, ni de ceux qui interviennent sur la scène nationale.

\section{Terre et conflits}

Les conflits liés à l'utilisation des terres ont été étudiés au sein de l'IP7 sous des angles différents, et dans des contextes géographiques et historiques eux aussi assez éloignés les uns des autres. Là aussi, il est apparu très nettement que, pour comprendre les dynamiques de ces conflits et élaborer des stratégies d'intervention efficaces, l'on ne peut se contenter de faire le lien entre la question de l'accès à la terre, de sa gestion ou de son utilisation d'une part, et, de l'autre, les tensions éventuelles ou la violence pouvant en résulter. De nombreuses autres variables doivent être prises en compte.

Ainsi, en Ethiopie, la mise en œuvre de politiques publiques dans des domaines ne concernant pourtant pas directement la politique agraire ou foncière a eu une influence considérable sur l'utilisation des terres et sur les stratégies de (sur)vie au quotidien (livelihood strategies). C'est le cas, par exemple, des politiques de sécurité, de décentralisation administrative et de distribution de l'aide alimentaire, laquelle est intimement liée à la politique humanitaire au niveau international. Si l'on se penche par exemple sur ce dernier aspect, il ressort que la distribution continue d'aide alimentaire dans des régions pastorales va à l'encontre d'une gestion durable des ressources naturelles. Au gré de ces distributions, on assiste à des regroupements importants de populations en des lieux qui ne s'y prêtent pas forcément, et ces politiques contribuent d'une manière générale à l'accroissement de la population dans certaines zones, créant ainsi rapidement des déséquilibres importants et exerçant une forte pression sur les terres. De plus, ces distributions entraînent et entretiennent souvent des modes de consommation et d'utilisation des ressources naturelles tout à fait 
inadéquats ${ }^{28}$. Pour renverser la tendance, des réformes doivent être mises en œuvre dans le domaine du droit foncier en général et de la propriété en particulier. Or, ces droits sont ancrés dans des dynamiques sociales et historiques, et ils font sens dans des cadres de vie et des réseaux sociaux qui ne sauraient être modifiés du jour au lendemain. Par ailleurs, ils fonctionnent selon des logiques qui échappent aux politiques gouvernementales, en tout cas dans les territoires semi-arides des basses terres étudiés dans le cadre de l'IP7. Le fait que le gouvernement éthiopien ne dispose à ce jour d'aucune politique pastorale au sens propre en est une bonne indication. Cela rend en outre très difficile tout engagement à l'échelle nationale dans le domaine pastoral.

En amont de l'utilisation et de la mise à profit des terres, que ce soit dans le domaine de l'agriculture ou dans celui du pastoralisme, se pose bien entendu la question de leur distribution. C'est sur cet aspect, entre autres, que se sont concentrées, dans le cadre de l'IP7, les recherches menées à l'ESZ sur l'Asie du Sud (Inde et Bangladesh) et du Sud-Est (Philippines, Indonésie). Plus précisément, elles ont mis l'accent sur les rapports entre «colons» et populations «indigènes» dans l'accès à la propriété foncière, notamment dans les régions de frontière (au sens social aussi bien que géographique). Lorsque des colons s'installent dans ces régions, que ce soit à l'initiative de l'Etat ou de leur propre chef, il en résulte généralement des tensions entre ceux-ci et les communautés indigènes qui se sentent menacées par les nouveaux arrivants. Les conflits violents qui en découlent parfois se cristallisent le plus souvent autour de la question de l'«indigénité » et génèrent des discours et des pratiques anti-immigrants au nom du droit des «autochtones» à disposer de «leurs» terres. Dans ces cas également, il serait simpliste de réduire les dynamiques de ce type de conflits à la seule question de l'accès aux ressources foncières, ou à celle de leur manque suite à l'installation des colons. Là non plus, les conflits en question ne sont pas seulement «environnementaux»; il s'agit bien de constellations de conflits de différents ordres et d'origines diverses (ethniques, politiques, sociaux, économiques, culturels, etc.), et toute tentative de médiation dans ce genre de conflits se doit de prendre en compte cette complexité2 ${ }^{2}$.

L'exemple de l'attribution des terres (land titling) dans l'île de Mindanao, aux Philippines, met bien en lumière toute la complexité et l'ambivalence des stratégies d'intervention dans ce domaine. En 1997, le gouvernement philippin s'est doté, avec l'Indigenous Peoples Rights Act (IPRA), d'une loi inédite dans toute l'Asie du Sud-Est en ce qui concerne la protection des populations indigènes. En donnant aux communautés indigènes le droit de revendiquer - et d'obtenir - un certificat de propriété sur leurs terres, cette loi institue en effet un système apparemment efficace de protection des minorités indigènes. Toutefois, à y regarder de plus près, on constate que les choses ne sont pas si simples. L'IPRA s'inscrit en effet dans une stratégie hégémonique de l'Etat philippin qui cherche à étendre son contrôle dans des zones dites «de frontière», au sens où la présence de l'Etat y est

28 Voir C. Ammann, Nahrungsmittelhilfe in der Somali Region Äthiopiens 1983-2004. Eine Verteilungsanalyse, Working Paper, $n^{\circ} 8$, Center for Comparative and International Studies, Zürich, Universität Zürich und ETHZ, 2005.

29 Une partie de ces recherches, menées sous la direction de Jürg Helbling et Danilo Geiger, a été publiée dans D. Geiger (ed.), Frontier Encounters: Indigenous Communities and Settlers in Asia and Latin America, New Brunswick, NJ, Transaction Publishers, 2006. 
généralement très faible et son pouvoir fortement remis en cause, parfois par des groupes armés. Ce qui apparaît donc de prime abord comme une mesure performante de protection des minorités ethniques est en fait également, ou surtout, un instrument hégémonique dans les mains de l'Etat ${ }^{30}$.

Si les recherches menées par l'IP7 sur les conflits liés à l'eau ou à la terre ont montré quelque chose, il s'agit bien de l'importance primordiale du facteur humain dans le cadre de conflits prétendument «environnementaux». De ce fait, la prévention comme la résolution de tels conflits passent non pas par des interventions d'ordre technique visant à influer soit sur l'origine environnementale de ces conflits (lutte contre la sécheresse par exemple), soit sur la distribution ou la répartition des ressources (réseaux d'irrigation, distribution de l'eau et des terres), mais par des interventions relatives à la gestion humaine, sociale, politique, économique et culturelle desdites ressources. Cette approche anthropocentrée de la gestion et de l'utilisation des ressources, en mettant l'individu et le groupe social au centre de l'analyse, vise aussi à étudier les dimensions institutionnelles de ces conflits et à saisir le potentiel de prévention et de résolution qui se trouve dans ces institutions, qu'elles soient coutumières, communautaires ou étatiques.

\section{Perspectives: remettre "l'Etat au milieu de l'analyse»}

Les conflits restent principalement, par définition, des interactions entre humains. Si le contexte change suite à des modifications climatiques ou autres, cela peut certes influencer les conditions-cadres des interactions humaines, mais il reste aux hommes et aux femmes à gérer leurs relations dans et avec cet environnement. La caractéristique première de ces conflits est donc qu'ils sont d'ordre social et non pas d'ordre environnemental. Cela ne veut pas dire qu'il faille négliger l'importance de conflits qui se développent autour de l'utilisation de ressources naturelles, mais il s'agit également de ne pas chercher trop rapidement de nouvelles explications univoques et réductrices à ce genre de phénomène. Même si les travaux de la fin des années 1990 avaient déjà souligné l'importance d'une analyse différenciée, prenant en compte différents facteurs et évitant toute causalité simpliste, il importe de souligner à nouveau ce point, notamment au vu des discussions apparues il y a quelques années sous le label greed versus grievance ${ }^{31}$ ainsi que sous celui des «nouvelles guerres $»^{32}$.

Avec leur thèse réductrice, revenant à dire qu'aujourd'hui la plupart des conflits dans le tiers-monde étaient dus davantage à l' «avidité» des «rebelles» qu'à des raisons politiques, Paul Collier et Anke Hoeffler ont attiré l'attention politique et scientifique sur le rôle des aspects économiques dans ce genre de conflits. Néanmoins, tout comme les conflits liés à l'environnement ne se réduisent pas à un «récit environnemental», remplacer le «récit politique» par un «récit économique» revient à fausser la perspective ${ }^{33}$. Il n'en demeure pas moins que, en

30 Voir à ce sujet I. Wenk, «Bounded Spaces of Coexistence: Land Titling and Settlers on Indigenous Domains in Mindanao, the Philippines », Tsantsa, $\mathrm{n}^{\circ}$ 10, 2005, pp. 181-185.

31 P. Collier, A. Hoeffler, Greed and Grievance in Civil War, Washington, DC, World Bank, 2000.

32 H. Münkler, Die neuen Kriege, Reinbek, Rowohlt, 2002.

33 Pour une analyse critique, parmi de nombreuses autres, des thèses de Paul Collier et Anke Hoeffler, voir R. Marchal, C. Messiant, «De l'avidité des rebelles. L'analyse économique de la guerre civile selon Paul Collier», Critique internationale, $\mathrm{n}^{\circ}$ 16, juillet 2002, pp. 58-69. 
mettant en avant les dimensions économiques, le débat lancé par Paul Collier et Anke Hoeffler comporte un lien intéressant avec nos recherches. Ces dernières traitent en effet aussi bien de la réglementation de la propriété et de son utilisation (property and user rights) que des liens entre économies locale, nationale et mondiale. Quant nous réfléchissons à des solutions liées à l'utilisation des ressources naturelles, il peut s'agir, outre d'intervenir sur le contexte politique, social et économique de la gestion de ces ressources, de trouver des formes de commercialisation ou de revenu alternatives pour les populations concernées. Nous en arrivons ainsi au rôle de différents régimes de commerce, aux alternatives potentielles telles que le commerce équitable, ou même aux contributions éventuelles d'entreprises multinationales. Il nous semble que ces principes économiques, ainsi que les régimes de propriété et les stratégies de pouvoir qui y sont attachés, ne sont pas suffisamment pris en compte par les programmes d'intervention occidentaux et qu'ils n'ont pas non plus fait l'objet de nombreuses recherches en rapport avec des conflits.

Par ailleurs, le rôle des institutions est crucial pour saisir le contexte de conflits liés à l'utilisation de ressources naturelles, mais aussi et surtout pour saisir le potentiel de transformation de tels conflits. Ce rôle a eu tendance à être sousestimé par la plupart des recherches antérieures, mais aussi par nos recherches lancées au début de la première phase du NCCR Nord-Sud. L'offre et la demande de ressources naturelles ne se développent en effet pas dans un vide; elles font partie d'un système de ressources plus large dans le cadre duquel des efforts de mitigation peuvent avoir lieu par le biais d'institutions. Cela vaut d'autant plus lorsqu'il s'agit de tensions ou de conflits internationaux, comme c'est souvent le cas en rapport avec les conflits liés à l'utilisation de l'eau. Le fait de savoir s'il y a effectivement abandon ou pénurie de ressources dépend du rôle et de la capacité de ces institutions $\mathrm{s}^{34}$, et elles sont d'autant plus aptes à résoudre ou à transformer d'éventuels conflits qu'elles établissent des liens entre différentes ressources en question et peuvent ainsi offrir des échanges ou des accords à travers une large base de ressources, qu'elles contiennent des mécanismes de monitoring pour les solutions trouvées et qu'elles sont ancrées dans un cadre institutionnel stable ${ }^{35}$.

Finalement, l'étude de conflits liés à l'utilisation des ressources naturelles touche au fondement même des Etats dans lesquels ils se déroulent. Premièrement, bien souvent les ressources en question et les modes de vie qui en découlent constituent la base vitale politique et économique des Etats concernés. Les dynamiques historiques et politiques de construction des Etats sont elles-mêmes indissociables de la disponibilité de ces ressources et de leur contrôle, tout comme l'ont été, par exemple, l'accès au pouvoir politique et la formation d'une classe dirigeante au sortir de l'époque coloniale en Afrique. Deuxièmement, les modes de gestion des droits de propriété ou des conflits liés à l'utilisation de ces ressources, le contrôle et la mise en œuvre de tels droits et la gestion des procédures sont étroitement liés au pouvoir et au mode de fonctionnement de l'Etat. Toute réforme ou transformation de ces «acquis» représente potentiellement une réforme ou une refonte de l'Etat lui-même. C'est pourquoi, en commençant

\footnotetext{
34 M.F. Giordano, M.A. Giordano, A.T. Wolf, «International Resource Conflict and Mitigation », Journal of Peace Research, vol. 42, n 1, 2005, pp. 47-65.

35 Ibid., pp. 59-61.
} 
par analyser des conflits locaux liés à l'utilisation des terres et à la répartition de l'eau, on arrive à des questions touchant l'essentiel du fonctionnement des systèmes de gouvernance aux différents échelons de l'Etat. Et, souvent, à ce niveau étatique viennent s'ajouter un échelon international constitué par les organisations internationales et un échelon transnational où fonctionnent les ONG nationales et internationales actives dans le pays en question.

En guise de conclusion, nous restons convaincus que la recherche sur les conflits liés à l'utilisation des ressources naturelles est une voie prometteuse. De par la nature de son sujet, elle a contribué à revaloriser le contexte géographique, mais aussi historique, social et économique des conflits étudiés. Toutefois, il importe à l'avenir d'ancrer davantage les recherches liées à ce type de conflits dans la réflexion apportée aux institutions de manière générale et au rôle de l'Etat et de ses différentes formes en particulier. Cela vaut d'autant plus que les nouvelles recherches, dont font également partie les nôtres, se concentrent sur les modes de gestion et de transformation de ces conflits. De telles analyses ne peuvent être élaborées de manière constructive en dehors du contexte politique. Cela vaut a fortiori pour la recherche d'éventuelles solutions et pour leur mise en œuvre. 